\title{
Message from the Steering Committee Chair
}

\section{QSIC 2005}

W ${ }_{\text {(QSIC 2005). }}^{\text {elcome to Melbourne, Australia, for the Fifth International Conference on Quality Software }}$

Software is playing an important role in our daily lives. Unfortunately, software systems often fail to deliver according to promises. Many popular systems are renowned for their user-friendliness but notorious for their unresolved faults. The QSIC conference provides a forum to bring together researchers and practitioners who work on improving the quality of software, enabling them to exchange ideas and present new results in this challenging area.

QSIC 2005 would not have been successful without the hard work of many volunteers. We are particularly grateful to T. Y. Chen of our Steering Committee for making this conference possible. We would like to thank General Chair Douglas Mitchell for his leadership, Program Co-Chairs KaiYuan Cai and Atsushi Ohnishi and their team for creating an excellent program that we shall enjoy for the next two days, and Organizing Committee Chair Yun Yang and his team for arranging the venue and social packages that we shall not forget. We are grateful to M. F. Lau, who serves not only as publicity chair to attract all of you here, but also as co-editor of the conference proceedings to ease the heavy workload of the program co-chairs. Special thanks are also due to Mary Lou Soffa of the University of Virginia for kindly agreeing to be our keynote speaker.

For the first time in the history of QSIC, we have four international workshops being held in parallel with the main conference. We should extend our appreciation to all the workshop co-chairs: Yun Yang, Volker Gruhn, S. C. Cheung, Jun Han, Fun Ting Chan, Laurence Dooley, Michael Winikoff, and Hong $\mathrm{Zhu}$, as well as their respective teams.

We should also thank all of you, who, as authors and participants, are helping us to assure the quality of this outstanding conference. We hope you will find this conference useful and enjoyable.

Our last two conferences were held in Dallas, Texas, USA, and Braunschweig, Germany. We are currently in Melbourne, Australia. It is natural for us to move back to Asia next year, so we are pleased to announce that QSIC 2006 will be held in Beijing, China. We look forward to seeing you again next year in the Forbidden City.

T. H. Tse The University of Hong Kong, Hong Kong 